\title{
COVID-19 in Philadelphia-negative myeloproliferative disorders: a GIMEMA survey
}

\author{
Massimo Breccia ${ }^{1}{ }^{1}$ - Alfonso Piciocchi ${ }^{2}$ - Valerio De Stefano $\mathbb{1}^{3}$ - Guido Finazzi ${ }^{4}$ - Alessandra lurlo ${ }^{5}$ Paola Fazi ${ }^{2}$. \\ Stefano Soddu ${ }^{2} \cdot$ Bruno Martino $^{6}$. Francesca Palandri $\Phi^{7}$. Sergio Siragusa ${ }^{8}$. Francesco Albano ${ }^{9}$. \\ Francesco Passamonti ${ }^{10} \cdot$ Marco Vignetti $^{1,2} \cdot$ Alessandro M. Vannucchi $\mathbb{1}^{11}$
}

Received: 3 August 2020 / Revised: 8 August 2020 / Accepted: 14 August 2020 / Published online: 25 August 2020

(c) The Author(s), under exclusive licence to Springer Nature Limited 2020

Persons with cancers are more likely to be affected by SARS-CoV-2 infection: it has been estimated that there is an increased risk of $1 \%$ [95\% CI 0.6-1.7\%] compared to $0.1 \%$ of normal [95\% CI $0-0.12 \%$ ] [1]. Several controversies on this topic emerged and the real potential risk in different cancer types remains unclear [2,3]. A cohort study conducted in two Chinese centers in Wuhan on 128 hospitalized hematological cancer patients showed that 13 (10\%) of them developed COVID-19 infection. No differences in baseline features were noted between subjects with hematological cancers that developed the infection and patients who did not. Among patients tested positive for COVID-19 (no patients with myeloproliferative disorders reported), the incidence was similar to normal healthcare providers $(7 \%)$ but the mortality rate was increased to $62 \%$ as compared to $0 \%$ of these latter category [4]. Recently, the Chinese group of Hubei reported also the prevalence of COVID-19 infection (0.9\%) in Philadelphia-positive chronic myeloid leukemia $(\mathrm{Ph}+\mathrm{CML})$ patients, that resulted ninefold higher than $0.1 \%$ reported in normal, but lower if compared to $10 \%$ in other hematological malignancies or $7 \%$ in healthcare providers [5]. The low rate of COVID-19

Massimo Breccia

breccia@bce.uniroma1.it

1 Department of Precision and Translational Medicine, Sapienza University, Rome, Italy

2 GIMEMA Foundation, Rome, Italy

3 Department of Radiological and Hematological Sciences, Section of Hematology, Catholic University, Fondazione Policlinico A. Gemelli IRCSS, Rome, Italy

4 Hematology and Bone Marrow Transplant Unit, ASST Papa Giovanni XXIII, Bergamo, Italy

5 Hematology Division, Foundation IRCCS Ca' Granda Ospedale Maggiore Policlinico, Milan, Italy infection in CML was recently confirmed in Italy [6]. At present, is still unclear the prevalence of COVID-19 infection in Philadelphia-negative myeloproliferative disorders (MPN) and the role of JAK2 inhibitor, ruxolitinib, a drug successfully used in myelofibrosis and polycythemia vera, implicated in modulation and reduction of cytokines release.

During the COVID-19 emergency, the GIMEMA group realized a cross-sectional survey with the aim to explore the prevalence of infection in $\mathrm{Ph}-\mathrm{MPN}$ in Italy. Survey data were collected and managed using the REDCap electronic data capture tools hosted at the GIMEMA Foundation [7]. The survey refers to the phase 1 of pandemic infection with a time lapse from the end of February to first day of April. Thirty-four centers compiled the survey, referring a whole cohort of $13.248 \mathrm{Ph}-\mathrm{MPN}$ patients regardless of ongoing therapies. Overall, 1095 patients were in treatment with ruxolitinib, 829 for myelofibrosis (75.7\%) and 266 because affected by polycythemia $(24.3 \%)$. During this period, 36 were found positive for COVID-19 (33.6\% of patients tested but $0.002 \%$ of the whole cohort).

6 Hematology Unit, Grande Ospedale Metropolitano "BianchiMelacrino-Morelli”, Reggio Calabria, Italy

7 Institute of Hematology "L. and A. Seràgnoli", St. OrsolaMalpighi University Hospital, Bologna, Italy

8 Haematology Unit, Department PROMISE, University of Palermo, Palermo, Italy

9 Department of Emergency and Organ Transplantation (D.E.T.O.), Hematology Section, University of Bari, Bari, Italy

10 Division of Hematology, Department of Medicine and Surgery, Ospedale di Circolo, University of Insubria, ASST Sette Laghi, Varese, Italy

11 CRIMM, Center Research and Innovation of Myeloproliferative Neoplasms, University of Florence, AOU Careggi, Florence, Italy 
Of the patients tested positive, $13(36 \%)$ were asymptomatic, 13 were affected only by flu-like symptoms (36\%), and 10 were affected by pneumonia related by COVID-19 ( $27.8 \%$ of positive cohort, $0.5 \%$ of the whole cohort). Two patients required noninvasive mechanical ventilation, whereas four positive patients required invasive ventilation. Eight positive patients died during this period of observation with a mortality rate of $22 \%$ (34\% of symptomatic patients). We also collected information about the incidence of pneumonia not related to COVID-19 during the same period: 66 patients were reported affected by not-related pneumonia and 6 patients negative for COVID-19 died $(9 \%)$

It has been reported that patients with severe COVID-19 infection showed lymphopenia and increased plasma concentrations of pro-inflammatory cytokines (IL6, IL10, TNFalpha, granulocyte-colony stimulating factor) and seems that IL6 levels were predictive for the severity of infection [8].

Among the possible drugs proposed against COVID-19, ruxolitinib, a JAK $1 / 2$ inhibitor, has been referred as one of the most promising drug. The drug can be prescribed for the treatment of myelofibrosis after the results of the randomized COMFORT trials $[9,10]$ in which determined an improvement of quality of life, reducing the spleen dimensions with relative increase in overall survival compared to best available therapy. The drug has been tested also in acute and chronic graft-versus-host disease refractory to other therapies, which determined 55\% of ORR [11]. Ruxolitinib decreases the secretion of pro-inflammatory cytokines, such as TNFa, IL1, IL6, and IFNg, and through the modulation of the STAT pathway, reduces the activity of Th lymphocytes [12]. For these reasons, 14 studies are actually ongoing in which ruxolitinib will be tested in patients with COVID-19 infection and cytokine storm with severe respiratory symptoms. In Italy, the Italian Medicines Agency AIFA authorized a compassionate use of the drug for patients with COVID-19 infection without the need of mechanical ventilation.

The results of this survey showed that the incidence of COVID-19 infection has proven low in $\mathrm{Ph}-\mathrm{MPN}$ patients, similar to the incidence observed in $\mathrm{Ph}+\mathrm{CML}[5,6]$. A certain protective role of JAK2 inhibitor cannot be excluded and prospective data on specific dataset will clarify its potential. Indeed, the mortality rate seems higher if compared to negative patients who developed non-COVID related pneumonia. A large retrospective/prospective cohort of $\mathrm{Ph}-\mathrm{MPN}$ COVID-19 positive patients should be analyzed in order to establish the effects of ruxolitinib and its real potentiality to moderate severity of respiratory symptoms. Moreover, how the pandemic emergency had an impact on daily management of MPN patients remains to be determined.

Acknowledgements The authors wish to thank all the GIMEMA centers that completed the survey.

\section{Compliance with ethical standards}

Conflict of interest The authors declare that they have no conflict of interest.

Publisher's note Springer Nature remains neutral with regard to jurisdictional claims in published maps and institutional affiliations.

\section{References}

1. Liang W, Guan W, Chen R, Wang W, Li J, Xu K, et al. Cancer patients in SARS-CoV-2 infection: a nationwide analysis in China. Lancet Oncol. 2020;21:335-7.

2. Xia Y, Jin R, Zhao J, Li W, Shen H. Risk of COVID-19 for patients with cancer. Lancet Oncol. 2020;21:e180.

3. Wang H, Zhang L. Risk of COVID-19 for patients with cancer. Lancet Oncol. 2020;21:e181.

4. He W, Chen L, Chen L, Yuan G, Fang Y, Chen W, et al. COVID19 in persons with haematological cancers. Leukemia. 2020;24: $1-9$

5. Li W, Wang D, Guo J, Yuan G, Yang Z, Gale RP, et al. COVID19 in persons with chronic myeloid leukaemia. Leukemia. 2020;34:1799-804.

6. Breccia M, Abruzzese E, Bocchia M, Bonifacio M, Castagnetti F, Fava C, et al. Chronic myeloid leukemia management at the time of the Covid-19 pandemic in Italy. A campus CML survey. Leukemia. 2020;34:2260-1.

7. Harris PA, Taylor R, Thielke R, Payne J, Gonzalez N, Conde JG. Research electronic data capture (REDCap) - a metadata-driven methodology and workflow process for providing translational research informatics support. J Biomed Inf. 2009;42:377-81.

8. Huang C, Wang Y, Li X, Ren L, Zhao J, Hu Y, et al. Clinical features of patients infected with 2019 novel coronavirus in Wuhan, China. Lancet. 2020;395:497-506.

9. Verstovsek S, Mesa RA, Gotlib J, Gupta V, DiPersio JF, Catalano $\mathrm{JV}$, et al. Long-term treatment with ruxolitinib for patients with myelofibrosis: 5-year update from the randomized, double-blind, placebo-controlled, phase 3 COMFORT-I trial. J Hematol Oncol. 2017;10:55.

10. Harrison CN, Vannucchi AM, Kiladjian JJ, Al-Ali HK, Gisslinger $\mathrm{H}$, Knoops L, et al. Long-term findings from COMFORT-II, a phase 3 study of ruxolitinib vs best available therapy for myelofibrosis. Leukemia. 2016;30:1701-7.

11. Zeiser R, von Bubnoff N, Butler J, Mohty M, Niederwieser D, Or $\mathrm{R}$, et al. Ruxolitinib for glucocorticoid-refractory acute graftversus-host disease. N Engl J Med. 2020;382:1800-10.

12. Elli EM, Baratè C, Mendicino F, Palandri F, Palumbo GA. Mechanisms underlying the anti-inflammatory and immunosuppressive activity of ruxolitinib. Front Oncol. 2019;9:1186. 